\title{
Interacciones entre hongos de la madera (Agaricomycete) y árboles nativos y exóticos de un ecosistema urbano (Córdoba, Argentina)
}

\author{
Interactions between wood-rotting fungi (Agaricomycetes) and native \\ and exotic trees from an urban ecosystem (Córdoba, Argentina)
}

\author{
Federico Heredia a*, Guillermo Morera a, Gerardo Robledo ${ }^{a}$ Luciano Cagnolo a, Carlos Urcelay a,b \\ *Autor de correspondencia: a Universidad Nacional de Córdoba, Instituto Multidisciplinario de Biología Vegetal (IMBIV), \\ CONICET, casilla de Correo 495, Córdoba-5000, Argentina, federicoheredia@live.com

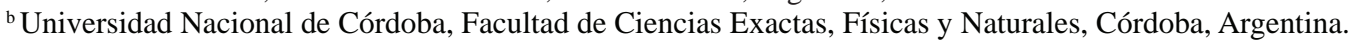

\begin{abstract}
SUMMARY
The replacement of natural areas by urban areas promotes changes in the composition of tree communities, frequently favoring the occurrence of exotic species. This may have direct implications in the structure of pathogenic fungal communities which grow in these hosts. Here we examine: a) the structure of the interaction networks between urban tree species and their pathogenic wood-decay fungi, b) the composition of pathogenic fungi in native and exotic hosts. The interactions network shows a nested pattern where several species of pathogenic fungi establish multiple interactions with various tree species, both native and exotic; while others establish more specific interactions. Richness of pathogenic fungi was higher in exotic trees than in natives. Thirteen fungal species occurred exclusively in exotic hosts, one in natives and the remaining nine species shared both exotic and native hosts.
\end{abstract}

Key words: plant-fungus interactions, interaction networks, nested topology, urban trees.

\section{RESUMEN}

El reemplazo de áreas naturales por zonas urbanizadas afecta la composición de las comunidades arbóreas, generalmente favoreciendo la ocurrencia de especies vegetales exóticas. Esto tendría implicancias directas en la estructuración de las comunidades de hongos patógenos que hospedan estas especies arbóreas. En este trabajo se propuso examinar: a) la estructura de la red de interacciones entre especies del arbolado urbano y los hongos patógenos degradadores de la madera, b) la composición de hongos patógenos en especies nativas y exóticas. Se observó que la red de interacciones mostró un patrón de anidamiento en donde varias especies de hongos patógenos establecen múltiples interacciones con diversas especies arbóreas, tanto nativas como exóticas, mientras que otras establecieron interacciones más específicas. Se observó mayor riqueza de hongos en árboles exóticos en comparación a sus pares nativos. Trece de las especies fúngicas se registraron exclusivamente en árboles exóticos, una exclusivamente en sustrato nativo y las nueve restantes compartieron tanto árboles exóticos como nativos.

Palabras clave: interacciones planta-hongo, redes de interacciones, topología anidada, arbolado urbano.

\section{INTRODUCCIÓN}

El movimiento de especies a gran escala (mayoritariamente animales y plantas) ha aumentado exponencialmente con la globalización del turismo y del comercio durante los dos últimos siglos promoviendo el establecimiento y expansión de organismos fuera de sus rangos de distribución originales; proceso conocido como invasiones biológicas. Sin embargo, en América Latina, el tema de las invasiones biológicas ha sido considerado relevante recién en las últimas décadas (Pauchard et al. 2011).

Existen diferentes mecanismos por los cuales las especies exóticas pueden convertirse en invasoras. Entre las más estudiadas pueden mencionarse los disturbios antrópicos que liberan recursos que son aprovechados por aquellas que presentan crecimiento rápido, los caracteres vegetativos y reproductivos diferentes a los de las nativas, que les permita aprovechar nichos disponibles, y las interacciones bióticas (Mack et al. 2000). Un ejemplo del último caso es el de las especies exóticas que escapan a los controles biológicos que poseen en sus rangos de distribución nativos. Específicamente, esta última hipótesis postula que las especies exóticas experimentan "liberación de enemigos naturales” tales como parásitos, patógenos y predadores, adquiriendo ciertas ventajas en relación a las especies vegetales nativas (Mitchell y Power 2003).

La flora urbana de las grandes ciudades presenta una gran proporción de especies exóticas que coexisten con especies vegetales nativas (Trentatovi et al. 2013). En este sentido, los ecosistemas urbanos proveen el escenario adecuado para comparar la composición de organismos antagonistas que, de algún modo, dependen de ellas y poner a prueba la hipó- 
tesis de que las especies arbóreas exóticas serían afectadas por una menor cantidad de hongos patógenos que las nativas.

Los hongos que degradan la madera de árboles vivos están considerados entre los principales patógenos de las especies arbóreas (Rajchenberg y Robledo 2013). A pesar de ser uno de los componentes fúngicos más importantes de los ecosistemas urbanos, no existen estudios que examinen específicamente las interacciones entre ellos y en especies arbóreas nativas y exóticas que coexisten en estos ecosistemas (Desprez-Loustau et al. 2007).

Las redes de interacción resultan una herramienta útil para el análisis de organismos que están relacionados de algún modo entre sí. Estas suelen representarse gráficamente mostrando el ensamble interactivo de una comunidad donde las especies se representan como nodos, y las interacciones entre éstas, como líneas o vínculos. Se reconocen dos patrones de organización básicos para las redes de interacción: anidados (i.e. cuando pocas especies establecen muchas interacciones con el resto de la red, y muchas otras establecen pocas, es decir, unas pocas especies generalistas y varias especialistas) y modulares (i.e. cuando ciertos grupos de especies establecen más interacciones dentro del mismo grupo, que con el resto de la red) (Lewinsohn et al. 2006). Recientemente se ha postulado que los hongos de la madera son más específicos del hospedador arbóreo de lo que se pensaba previamente (Robledo y Urcelay 2012, Rajchenberg y Robledo 2013). Por lo tanto, las comunidades de hongos cambiarían de acuerdo a la identidad y al origen biogeográfico de los árboles. Por ello cabe esperar que las comunidades de hongos asociadas a árboles exóticos y nativos difieran entre sí, además de presentar redes de interacción con topología modular (Vacher et al 2008).

El presente estudio se centra específicamente en las comunidades de hongos patógenos (Agaricomycetes, Basidiomycota) (Rajchenberg y Robledo 2013) que degradan la madera de árboles vivos de la Ciudad de Córdoba. En particular, se examina: a) la estructura de las redes de interacción entre especies del arbolado urbano y los hongos patógenos; b) la composición de hongos patógenos en especies nativas y exóticas.

\section{MÉTODOS}

Sitio de estudio. La ciudad de Córdoba es la segunda ciudad de Argentina con mayor número de habitantes (aproximadamente 1.500 .000 habitantes). Se encuentra a $440 \mathrm{~m}$ s.n.m. y se ubica en el faldeo oriental de las Sierras Chicas. Las precipitaciones medias anuales varían de 600 a $800 \mathrm{~mm}$, siendo el período de diciembre a febrero el más lluvioso. El arbolado urbano de la ciudad, reúne alrededor de 550.000 individuos distribuidos en todo el terreno municipal, incluyendo sitios privados y públicos ${ }^{1}$.

Francisco García Montaño. Ex jefe de Departamento de Agronomía de la Dirección de Espacios Verdes de la Municipalidad de Córdoba. Argentina. Comunicación personal.
Estudio de los especímenes. Se analizó un total de 80 colecciones de hongos patógenos depositadas en herbario del Museo Botánico, Facultad de Ciencias Exactas, Físicas y Naturales, Universidad Nacional de Córdoba (CORD), así como 20 nuevas colecciones que se obtuvieron en muestreos realizados fundamentalmente entre diciembre y mayo de los años 2011 y 2012. En total se analizaron 23 especies de hongos patógenos degradadores de la madera en 28 especies de árboles (cuadro 1). El total de las colecciones se obtuvo mediante muestreos oportunistas, realizados en distintos sectores de la ciudad (predominantemente Ciudad Universitaria y Parque Sarmiento), en donde cada colección registrada corresponde a un individuo arbóreo diferente $(\mathrm{n}=100)$.

Los basidiomas (cuerpos de fructificación y estructuras visibles en los hongos pertenecientes a la Clase Agaricomycetes del Phylum Basidiomycota) fueron colectados en troncos y ramas de árboles en pie vivos. Se realizó la identificación de cada ejemplar con el auxilio de microscopio Nikon eclipse E200 mediante la realización de cortes a mano alzada, y posteriormente montados en $\mathrm{KOH} 3 \%$ con floxina y en reactivo de Melzer. Los materiales coleccionados en los muestreos fueron depositados en el herbario del Museo Botánico, Facultad de Ciencias Exactas, Físicas y Naturales, Universidad Nacional de Córdoba (CORD).

Análisis de datos. Se consideraron como especies arbóreas nativas aquellas que son originarias de la región central y centro-norte de Argentina (Zuloaga et al. 2008), incluyendo algunas que no lo son específicamente de la ciudad Córdoba. Esto se debe a la alta capacidad de dispersión de los hongos, por lo cual su ocurrencia no estaría limitada en dichas especies arbóreas del ecosistema estudiado. Las consideradas exóticas son originarias de otros continentes.

Se construyó una red de interacciones para analizar las propiedades estructurales de la interacción entre la comunidad arbórea y la comunidad fúngica. Para ello se utilizó el paquete bipartite (Dormann et al. 2008) del programa estadístico R (R Development Core Team 2007). Este tipo de redes puede adoptar diferentes configuraciones básicas: formas de gradiente con una estructura de compartimientos (modular) o una estructura anidada (Lewinsohn et al 2006). Para conocer si las relaciones entre hongos patógenos y sustrato arbóreo están definidas al azar o responden a ciertas restricciones biológicas que las definan, se evaluaron los niveles de modularidad y anidamiento en la topología de la red, y se compararon los valores observados con los obtenidos a partir de 100 simulaciones generadas por un modelo nulo, que conserva el número de especies, interacciones y sumas marginales de la matriz de interacciones observadas (Dormann et al. 2008). Para comparar la riqueza de hongos patógenos entre especies nativas y exóticas se realizó un análisis de la varianza donde se incluyó la abundancia relativa de cada especie arbórea como covariable. En virtud de tomar ventaja del material colectado durante años y luego depositado en herbario, no se pudo 
Cuadro 1. Especies arbóreas estudiadas. Para cada especie se detalla el orden y familia a la cual pertenecen, además de la abundancia relativa calculada en base a datos obtenidos de Ciudad Universitaria (Re et al. 2011) (cantidad de individuos de cada especie / número total de individuos arbóreos, distribuidos en Ciudad Universitaria, $\mathrm{n}=8.872$ ).

Studied tree species. The order and family are specified for each one. The relative abundance of each species is also provided based on data obtained in Ciudad Universitaria (Re et al. 2011) (number of individuals of each species / total tree individuals distributed in Ciudad Universitaria, $\mathrm{n}=8$,872).

\begin{tabular}{|c|c|c|c|c|}
\hline Especie arbórea & Nombre científico & Estatus & Orden/Familia & $\begin{array}{c}\text { Abundancia } \\
\text { relativa }\end{array}$ \\
\hline Fresno & Fraxinus americana L. & Exótica & Lamiales/ Oleaceae & 0,0948 \\
\hline Olmo & Ulmus pumila L. & Exótica & Rosales/ Ulmaceae & 0,0833 \\
\hline Eucaliptus & Eucalyptus sp & Exótica & Myrtales/ Myrtaceae & 0,0635 \\
\hline Álamo & Populus sp & Exótica & Malpighiales/ Salicaceae & 0,0417 \\
\hline Mora & Morus sp & Exótica & Rosales/ Moraceae & 0,0406 \\
\hline Paraíso & Melia azedarach L. & Exótica & Sapindales/ Meliaceae & 0,0398 \\
\hline Prunus & Prunus sp & Exótica & Rosales/ Rosaceae & 0,0358 \\
\hline Siempre verde & Ligustrum lucidum W.T. Aiton & Exótica & Lamiales/ Oleaceae & 0,0266 \\
\hline Plátano & Platanus acerifolia (Aiton) Wild. & Exótica & Proteales/ Platanaceae & 0,0078 \\
\hline Roble sedoso & Grevillea robusta A. Cunn. ex R. Br. & Exótica & Proteales/ Proteaceae & 0,0054 \\
\hline Acacia blanca & Robinia pseudoacacia L. & Exótica & Fabales/ fabaceae & 0,0048 \\
\hline Roble & Quercus sp & Exótica & Fagales/ Fagaceae & 0,0039 \\
\hline Crespón & Lagerstroemia indica L. & Exótica & Myrtales/ Lythraceae & 0,0011 \\
\hline Aromo francés & Acacia dealbata Link & Exótica & Fabales/ Fabaceae & 0,0009 \\
\hline Sauce llorón & Salix babylonica L. & Exótica & Malpighiales/ Salicaceae & 0,0009 \\
\hline Algarrobo europeo & Ceratonia silicua L. & Exótica & Fabales/ Ceratoniaceae & 0,0001 \\
\hline Sofora & Maclura pomifera (Raf.) C. K. Schneid. & Exótica & Rosales/ Moraceae & - \\
\hline Grataegus & Pyracantha sp M. Roem. & Exótica & Rosales/ Rosaceae & - \\
\hline Aguaribay & Schinus areira L. & Nativa & Sapindales/ Anacardiaceae & 0,0034 \\
\hline Jacarandá & Jacaranda mimosifolia D. Don & Nativa & Lamiales/ Bignoniaceae & 0,1514 \\
\hline Palo borracho & Chorisia sp & Nativa & Malvales/Malvaceae & 0,0418 \\
\hline Visco & Acacia visco Lorentz ex Griseb. & Nativa & Fabales/ Fabaceae & 0,0112 \\
\hline Algarrobo & Prosopis sp & Nativa & Fabales/ Fabaceae & 0,0099 \\
\hline Molle & Lithraea molleoides (Vell.) Engl. & Nativa & Sapindales/ Anacardiaceae & 0,0020 \\
\hline Tala & Celtis ehrenbergiana (Klotzsch) Liebm. & Nativa & Rosales/ Cannabaceae & 0,0017 \\
\hline Chañar & Geoffroea decorticans (Gillies ex Hook. et Arn.) Bukart & Nativa & Fabales/ Fabaceae & 0,0009 \\
\hline Sauce criollo & Salix humboldtiana Wild. & Nativa & Malpighiales/ Salicaceae & 0,0007 \\
\hline Espinillo negro & Acacia atramentaria Benth. & Nativa & Fabales/ Fabaceae & 0,0001 \\
\hline
\end{tabular}

controlar el esfuerzo de muestreo realizado sobre cada especie de árbol. Dado que los materiales fueron colectados principalmente por los autores con la atención puesta en los hongos, independientemente de si estaban en árboles nativos o exóticos, se asume que no hubo un esfuerzo de muestreo particular hacia árboles nativos o exóticos, al menos de modo intencional. Además, si bien los valores de abundancia relativa de las especies para la ciudad no se encuentran disponibles, aquí utilizamos como referencia un reciente censo exhaustivo sobre la abundancia de cada especie de árbol realizado en Ciudad Universitaria (Re et al. 2011) que constituyó el principal área de muestreo en este estudio. Dicha covariable representa una variable aleatoria continua cuyo valor varía por cada unidad experimental y podría estar relacionada con la variable de respuesta. En este caso, la abundancia relativa de las especies arbóreas podría incidir en el número de especies de hongos patógenos, haciendo que la mayor densidad de una determinada especie arbórea en el ecosistema urbano, incremente las posibilidades de encontrar mayor cantidad de basidiomas de hongos patógenos. Debido a que la variable riqueza no mostró distribución normal, se realizó una trasformación a $\log _{10}$, cumpliendo de este modo con los supuestos del análisis. 


\section{RESULTADOS}

El análisis de redes mostró que la topología de las relaciones hongos patógenos-árboles resultó más anidada que lo esperable por azar (temperatura observada $=8,399$; temperaturas esperadas $>15, P<0,05$ ) (figuras 1 y 2 ) mientras que los niveles de modularidad no difirieron de lo esperable por azar ( $M$ observada $=0,580, M$ media esperada $=0,580, P>0,05)$. Al analizar las redes que involucran árboles nativos y exóticos por separado, no se observaron diferencias con lo esperable por azar, tanto para anidamiento como para modularidad $(P>0,05)$ en ambos casos).

La riqueza de hongos patógenos fue mayor en árboles exóticos en comparación a sus pares nativos $(F=9,38$, $P=0,001$ ) (figura 3 ). La abundancia relativa de las especies arbóreas no fue significativa en la comparación de riqueza de hongos degradadores de la madera $(F=1,17$; $P=0,291)$.

El número de especies arbóreas afectadas por los diferentes patógenos difiere según la especie de hongo analizada. Entre las especies fúngicas encontradas se hallaron trece exclusivamente en sustratos exóticos, una especie exclusivamente en sustrato nativo y las restantes nueve especies compartieron tanto sustrato exótico como nativo (figura 4).

\section{DISCUSIÓN}

Este estudio constituye un aporte sobre las relaciones entre los hongos patógenos y los hospedadores arbóreos nativos y exóticos que coexisten en un ecosistema urbano. Contrario a la predicción, la riqueza de especies fúngicas fue mayor en árboles exóticos que en nativos y esto no parece estar relacionado con sus abundancias relativas en el área de estudio. Esto implica también que cada hospedador nativo establece un menor número de relaciones con especies de hongos patógenos y que las relaciones establecidas con los hospedadores exóticos son más numerosas (figuras 1 y 4). De esta manera, las especies fúngicas presentes en sustrato exótico serían más generalistas que las que se presentan en árboles nativos. Esto sugiere que varios de los hospedadores arbóreos exóticos no experimentarían "liberación de enemigos naturales" en el área de estudio. Una hipótesis alternativa reciente postula que las plantas exóticas podrían estar propensas a experimentar “acumulación de patógenos" en sus rangos de distribución no nativos (Flory y Clay 2013). Unos de los factores que promovería la acumulación de patógenos es la alta densidad de los hospedadores. Si bien aquí observamos que la abundancia relativa de la especies arbóreas no se relacionó

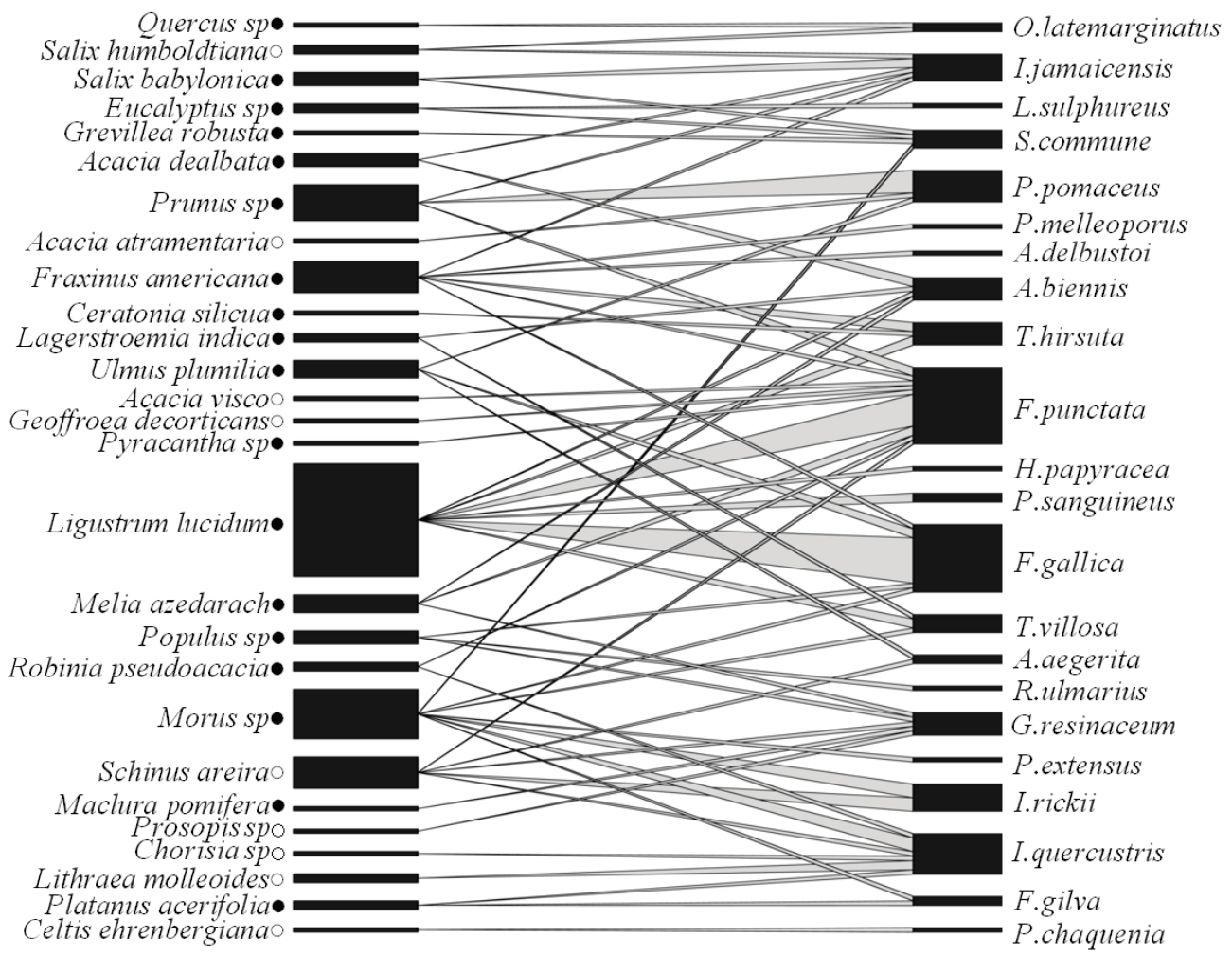

Figura 1. Red de interacción entre hongos patógenos (a la derecha) y especies arbóreas (a la izquierda; en blanco las especies nativas, en negro las especies exóticas). Las bandas negras indican la abundancia de las especies consideradas, y el ancho de línea de cada interacción refleja la frecuencia con la que ambas especies se relacionan.

Network interaction analyses, between pathogenic polypores (right) and tree species (on the left; in white native species, alien species in black). Black bars indicate the abundance of the species considered, and the line width of each interaction reflects the frequency with which both species are related. 


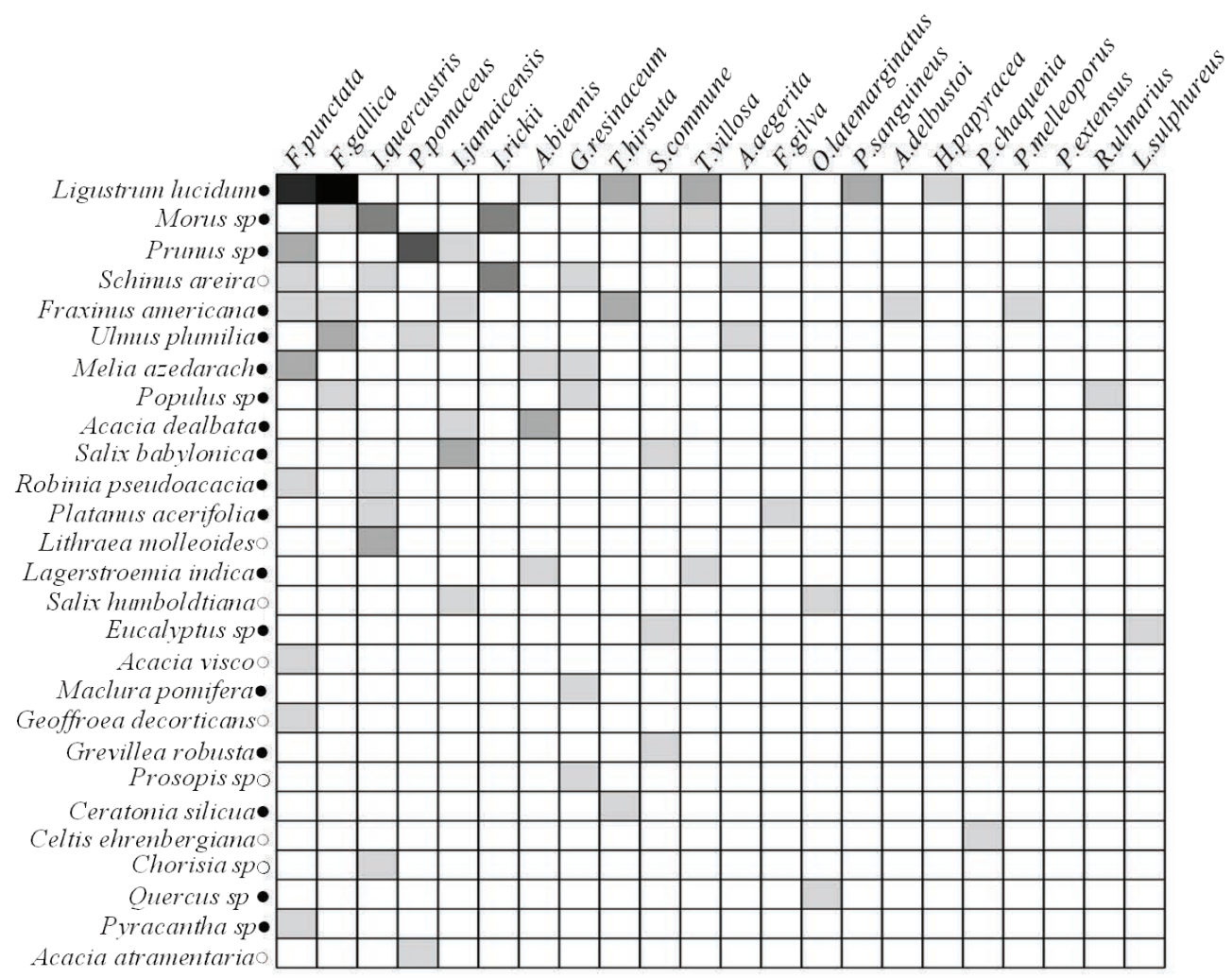

Figura 2. Análisis de anidamiento entre las especies arbóreas (en blanco las especies nativas, en negro las especies exóticas) y los hongos patógenos. La relación entre ambas especies, verifica este patrón (temperatura observada $=8,399$, temperaturas esperadas $>$ $15, * P<0,05)$.

Nested analyses of urban tree species (native species: white, alien species: black) and pathogenic polypores. The relationship between the two species confirms this pattern (observed temperature $=8.399$, expected temperatures $>15, P<0.05$ ). The temperature indicates the degree of disorder in the original data matrix, taking values ranging from $0^{\circ}$ (perfectly nested model) to $100^{\circ}$ (not nested model).

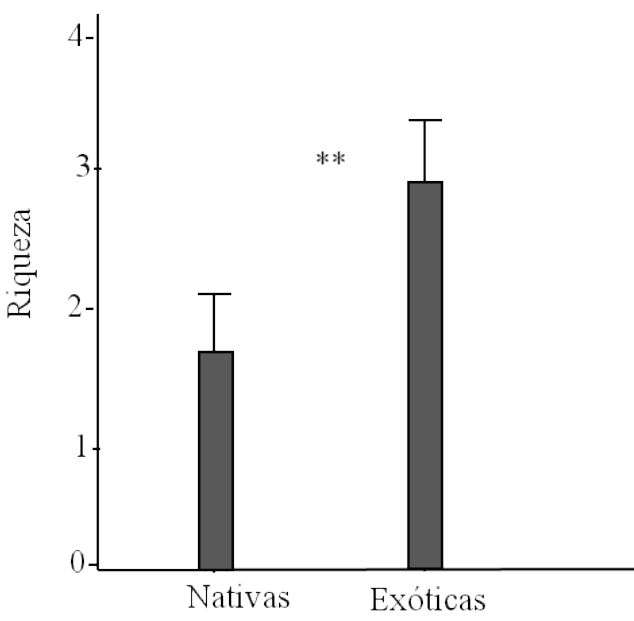

Figura 3. Comparación de la riqueza de hongos patógenos en árboles nativos del centro y norte argentino y exóticos presentes en la Ciudad de Córdoba. Se encontraron diferencias significativas entre ambos tipos de sustratos $(F=9,38 ; P=0,001)$.

Comparison of the richness of polypores pathogens in native and exotic trees from Córdoba city. Significant differences were found between the two types of substrates $(F=9.38 ; P=0.001)$. con la riqueza promedio de hongos, las especies exóticas que acumulan mayor cantidad de hongos patógenos en nuestro estudio (L. ludcidum y Morus sp.) se encuentran entre las más abundantes a nivel regional (centro de Argentina, incluyendo particularmente áreas adyacentes a la ciudad de Córdoba) (Giorgis et al. 2011).

Desde el punto de vista de los hongos, la red de interacciones muestra un patrón de anidamiento en donde varias especies de hongos patógenos establecen múltiples interacciones con diversas especies arbóreas, tanto nativas como exóticas (figura 1). Este suele ser el caso de los hongos patógenos más frecuentes mientras que los menos frecuentes parecen ser más específicos. En estos casos la evidencia sugiere que las interacciones establecidas entre esos hongos patógenos y sus hospedadores no implicarían mayores restricciones y que la asociación existente entre ambos tipos de entidades se debería tan solo a la probabilidad de encuentro entre ellas (figura 2). Sin embargo, este resultado contrasta con lo encontrado por Vacher et al. (2008) en bosques de Francia, donde la red de interacciones mostró una topología modular, asociada a la filogenia de las especies de plantas. El resultado del presente estudio 


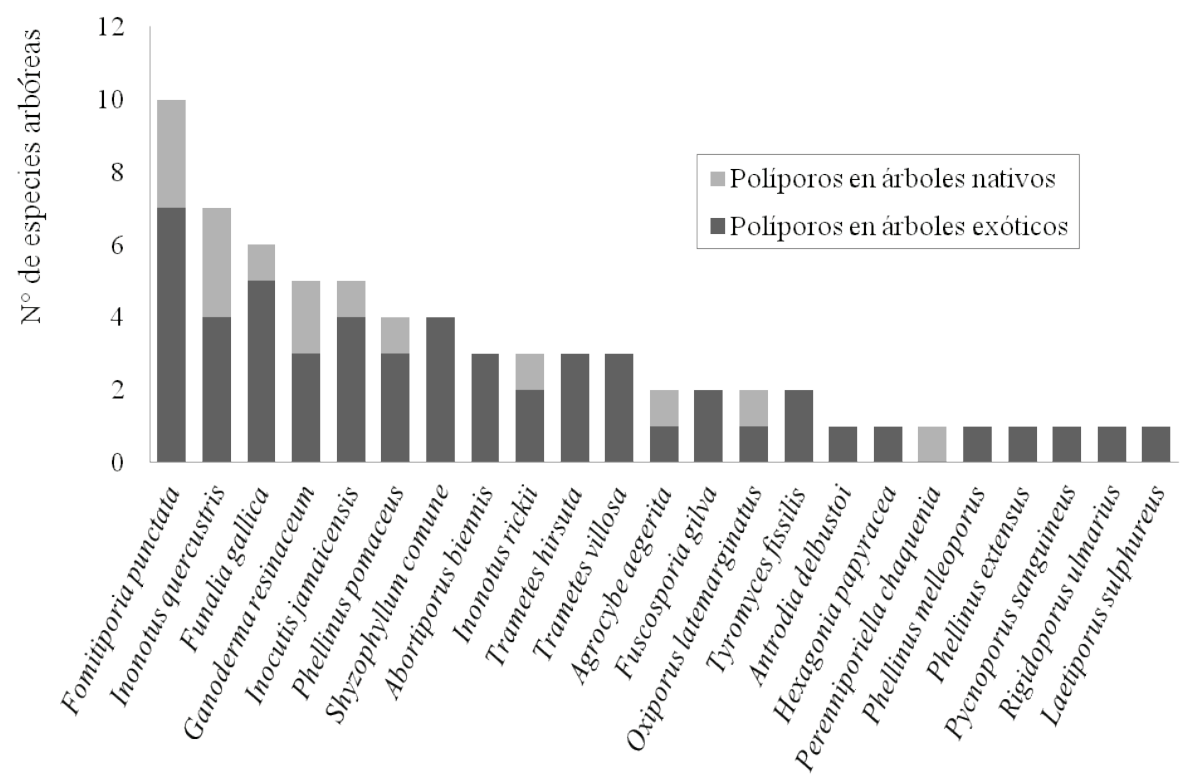

Figura 4. Hongos patógenos vs número de especies arbóreas registradas, caracterizadas según su condición exótica-nativa. Pathogenic polypores vs. registered number of tree species, characterized by exotic-native status.

podría ser consecuencia de interacciones que se presentan en un ambiente urbano donde co-ocurren especies que no se encontrarían en ambientes naturales. Sin embargo, resolver esta incógnita requiere replicar estudios de este tipo en otras ciudades y ambientes naturales.

Gran parte de las especies de hongos patógenos encontradas serían más generalistas de lo supuesto inicialmente (ver más adelante sobre la identificación de los hongos) y colonizan indistintamente tanto árboles nativos como exóticos. Estas especies de hongos generalistas suelen ser también las más abundantes (figura 4) por lo que serían las principales responsables de los procesos de degradación de la madera de árboles vivos en el ecosistema urbano (Urcelay y Robledo 2004).

Es importante poner en relieve, no obstante, que algunas especies de hongos patógenos (caracterizadas morfológicamente) aparentemente generalistas, pueden tratarse de complejos de especies donde cada una de ellas podría mostrar cierta especificidad por el hospedador arbóreo. Tal caso ha sido recientemente sugerido para Fomitiporia punctata (Robledo y Urcelay 2009, Urcelay et al. 2012, Rajchenberg y Robledo 2013), una de las especies que fue observada en varios hospedadores arbóreos. Estudios recientes han arrojado árboles filogenéticos del género $F o-$ mitiporia que permiten identificar clados bien definidos, cada uno constituido por especies de la misma región biogeográfica: 'Pantemplados', 'Indomalayos', 'paleotropicales (Africanos)' y Neotropicales (Sudamericanos) (Decock et al. 2007, Amalfi et al 2010). En el clado 'Neotropical' se ubican las colecciones de Fomitiporia presentes en árboles nativos del centro de Argentina estudiadas hasta el momento (por ejemplo, Condalia montana, Sebastiana commersoniana, Larrea divaricata, Bulnesia retamo, entre otras, ver Decock et al. 2007, Robledo y Urcelay 2009). Sin embargo, se han observado numerosas colecciones de este complejo sin diferencias morfológicas claras entre las citadas para árboles nativos y las observadas en árboles exóticos originarias de distintas regiones del mundo (por ejemplo, Robinia pseudoacacia y Fraxinus americana Pantempladas- Ligustrum lucidum y Melia azedarach -Indomalayas, ver Urcelay et al. 2012). Estudios moleculares serán necesarios para poner explícitamente a prueba esta hipótesis y revelar eventualmente la existencia de especies conocidas como crípticas o corroborar el carácter generalista de dichos hongos.

Más allá del posible carácter generalista de ciertas especies, algunas se presentaron exclusivamente en hospedadores exóticos (e.g. L. sulphureus). Se trataría, entonces, de especies de hongos originarias de otras regiones del globo con cierta especificidad que habrían llegado aquí por dispersión de las esporas (Hallenberg et al. 2001) o mediadas por la actividad antrópica (e.g. importación de maderas colonizadas por los patógenos). Llamativamente, algunas combinaciones de hongos patógenos y especies arbóreas exóticas no han sido observadas en los ecosistemas donde esos hospedadores arbóreos son nativos. Tal es el caso de los árboles Ligustrum lucidum y Morus spp. que muestran en este estudio mayor riqueza de hongos patógenos que las observadas en su rango de distribución nativo (China y Asia, respectivamente) (Dai 2012). Esto evidencia que la ocurrencia de hongos patógenos y hospedadores arbóreos fuera de sus rangos de distribución nativos, pueden generar nuevas combinaciones interactivas. Esto implica que no se puede descartar la posibilidad de que estos patógenos 
puedan en algún momento colonizar especies nativas (Loo 2009) generando así consecuencias impredecibles para las comunidades arbóreas nativas.

Algunos hongos patógenos hallados en este trabajo ( $G$. resinaceum e I. rickii) han sido mencionados como importantes agentes de pudrición en árboles en otro ecosistema urbano (Ciudad de Buenos Aires, Argentina), particularmente en individuos del árbol Platanus acerifolia (Robles et al. 2011). Si bien estas dos especies patógenas no han sido encontradas para dicho hospedador en este estudio, se las ha observado degradando la madera en otras especies arbóreas, tanto nativas como exóticas, fortaleciendo la idea de que se trataría de especies patógenas poco específicas.

Se ha observado que la producción de basidiomas se relaciona positivamente con la disponibilidad de sustrato, lo que sugiere que la presencia y número de basidiomas puede ser un buen indicador de la biomasa de micelio vegetativo que le da origen (Urcelay y Robledo 2009). Así, es frecuente que estudios similares al presentado aquí, empleen la observación de basidiomas para el estudio de las comunidades de hongos de la madera (Barrico y Azul 2012, Robles et al. 2011, por mencionar algunos recientes). Sin embargo, la ausencia de dichos cuerpos de fructificación no garantiza que la fase vegetativa del hongo esté ausente en el sustrato. Esta limitación metodológica podría ser subsanada a partir del empleo de herramientas moleculares que analicen el micelio vegetativo que efectivamente está degradando el sustrato (Ovaskainen et al. 2013). No obstante, es importante señalar que más allá de algunas diferencias en la detección de algunas especies, el uso de herramientas moleculares para la identificación de micelio vegetativo en el sustrato arroja conclusiones similares a las del análisis de las comunidades de hongos a partir de la ocurrencia de basidiomas (Ovaskainen et al. 2013). Por lo tanto, la aproximación a través del estudio de basidiomas sería adecuada, al menos, para tener una idea inicial sobre este tipo de interacciones.

\section{CONCLUSIONES}

El presente estudio muestra que las interacciones hongo patógeno-árbol serían de tipo especialista pero las especies fúngicas más abundantes establecen relaciones de tipo generalista, incluyendo árboles nativos y exóticos, lo que da como resultado una red de interacciones con una topología anidada. Los resultados muestran mayor riqueza de hongos patógenos en árboles exóticos en comparación con los nativos. Esto sugiere que los árboles exóticos del ecosistema urbano estudiado no estarían "liberados de los enemigos naturales" cuando crecen fuera de su rango de distribución nativo.

\section{AGRADECIMIENTOS}

Los fondos para el financiamiento de este trabajo fueron otorgados por MINCyT Córdoba, Programa PROTRI y FONCyT-PICT. G. Robledo, L. Cagnolo y C. Urcelay son investigadores del CONICET y agradecen el apoyo del Consejo Nacional de Investigaciones Científicas y Técnicas (CONICET) y la Universidad Nacional de Córdoba por haber provisto las facilidades para realizar este trabajo.

\section{REFERENCIAS}

Amalfi M, P Yombiyeni, C Decock. 2010. Fomitiporia in sub-Saharan Africa: morphology and multigene phylogenetic analysis support three new species from the Guineo-Congolian rainforest. Mycologia 102: 1303-1317.

Barrico L, A Azul. 2012. Biodiversity in urban ecosystems: Plants and macromycetes as indicators for conservation planning in the city of Coimbra (Portugal). Landscape and Urban Planning 106(1): 88-102.

Dai YC. 2012. Polypore diversity in China with an annotated checklist of Chinese polypores. Mycoscience 53(1): 49-80.

Decock C, S Herrera Figueroa, GL Robledo, G Castillo. 2007. Fomitiporia punctata (Basidiomycota, Hymenochaetales) and its presumed taxonomic synonyms in America: taxonomy and phylogeny of some species from tropical / subtropical area. Mycologia 99: 733-752.

Desprez-Loustau ML, C Robin, M Buee, R Courtecuisse, J Garbaye, F Suffert, DM Rizzo. 2007. The fungal dimension of biological invasions. Trends in Ecology Evolution 22(9): 472-480.

Dormann C, B Gruber, J Frund. 2008. Introducing the bipartite package: analyzing ecological networks. R news 8 (2): 8-11.

Flory SL, K Clay. 2013. Pathogen accumulation and long-term dynamics of plant invasions. Journal of Ecology 101(3): 607-613.

Giorgis MA, AM Cingolani, F Chiarini, J Chiapella, G Barboza, LA Espinar, M Cabido. 2011. Composición florística del Bosque Chaqueño Serrano de la provincia de Córdoba, Argentina. Kurtziana 36(1): 9-43.

Hallenberg N, N Kuffer. 2001. Long-distance spore dispersal in wood-inhabiting. Basidiomycetes 21(4): 431-436.

Lewinsohn TM, PI Prado, P Jordano, J Bascompte, JM Olesen. 2006. Structure in plant-animal interaction assemblages. Oikos 113: 174-184.

Loo JA. 2009. Ecological impacts of non-indigenous invasive fungi as forest pathogens. Biological Invasions 11(1): 81-96.

Mack RN, D Simberloff, W Mark Lonsdale, H Evans, M Clout, FA Bazzaz. 2000. Biotic invasions: causes, epidemiology, global consequences, and control. Ecological Applications 10(3): 689-710.

Mitchell CE, AG Power. 2003. Release of invasive plants from fungal and viral pathogens. Nature 421: 625-627.

Ovaskainen O, D Schigel, H Ali-kovero, P Auvinen, L Paulin. 2013. Combining high-throughput sequencing with fruit body surveys reveals contrasting life-history strategies in fungi. ISME Journal 7(9): 1-14.

Pauchard A, RA García, B Langdon, N Fuentes.2011. The Invasion of Non-Native Plants in Chile and their Impacts on Biodiversity: History, Current Status, and Challenges for Management. In Biodiversity Conservation in the Americas: Lessons and Policy Recommendations. Santiago de Chile, Chile. Editorial FEN-Universidad de Chile. p. 133-165.

Rajchenberg M, G Robledo. 2013. Pathogenic polypores in Ar- 
gentina. Forest Pathology 43(3): 171-184.

Re GE, C Eynard, M Martiarena, JM Menna, E Hick, M Gil. 2011. Los árboles de Ciudad Universitaria. Córdoba, Argentina. Universidad Nacional de Córdoba. 70 p.

Robledo G, C Urcelay. 2009. Hongos de la madera en árboles nativos del centro de la Argentina. Cordoba, Argentina. Universidad Nacional de Córdoba. 224 p.

Robledo G, C Urcelay. 2012. Mycogeography of south american polypores: substrate specificity and functional types to understand fungal distributions. In Anales de $63^{\circ}$ Congreso Nacional de Botânica (Brasil). 10 p.

R Development Core Team. 2007. R: A Language and Environment for Statistical Computing. R Foundation for Statistical Computing, Vienna, Austria. Consultado 18 dic. 2013. Disponible en http://www.R-project.org

Robles CA, CC Carmarán, SE Lopez. 2011. Screening of xylophagous fungi associated with Platanus acerifolia in urban landscapes: Biodiversity and potential biodeterioration. Landscape and Urban Planning 100(1-2): 129-135.
Trentanovi G, M Von der Lippe, T Sitzia, U Ziechmann, I Kowarik, A Cierjacks. 2013. Biotic homogenization at the community scale: disentangling the roles of urbanization and plant invasion. Diversity and Distributions 19(7): 738-748.

Urcelay C, G Robledo. 2004. Community structure of polypores (Basidiomycota ) in Andean alder wood in Argentina : Functional groups among wood-decay fungi ? Austral Ecology 29: 471-476.

Urcelay C, G Robledo, F Heredia, G Morera, F García Montaño. 2012. Hongos de la madera en el arbolado urbano de Córdoba. Córdoba, Argentina. Instituto Multidisciplinario de Biología Vegetal (UNC-CONICET). 104 p.

Vacher C, D Piou, ML Desprez-Loustau. 2008 Architecture of an Antagonistic Tree/Fungus Network: The Asymmetric Influence of Past Evolutionary History. Plos One 3: e1740.

Zuloaga FO, O Morrone, MJ Belgrano. 2008. Catálogo de las plantas vasculares del Cono Sur (Argentina, Sur de Brasil, Chile, Paraguay y Uruguay). Missouri, USA. Monographs of the Missouri Botanical Garden Press 107. 3486 p.

Recibido: 06.02.14

Aceptado: 09.09.14 